\title{
Variable Stellar Object Detection and Light Curves from the Solar Mass Ejection Imager (SMEI)
}

\author{
R. A. Hounsell ${ }^{1}$, M. F. Bode ${ }^{1}$, M. J. Darnley ${ }^{1}$, D. J. Harman ${ }^{1}$, \\ P. P Hick $^{2}$, A. Buffington ${ }^{3}$, B. V. Jackson ${ }^{3}$, J. M. Clover ${ }^{3}$ \\ and A. W. Shafter ${ }^{4}$ \\ ${ }^{1}$ Astrophysics Research Institute, Liverpool JMU, Birkenhead, CH41 1LD, UK \\ email: rah@astro.livjm.ac.uk \\ ${ }^{2}$ San Diego Supercomputer Center, University of California, La Jolla, CA 92093, USA \\ ${ }^{3}$ Center for Astrophysics and Space Sciences, Univ. of California, La Jolla, CA 92093, USA \\ ${ }^{4}$ Department of Astronomy, San Diego State University, San Diego, CA 92182, USA
}

\begin{abstract}
With the advent of surveys such as the Catalina Real-Time Transient Survey, the Palomar Transient Factory, Pan-STARRS and GAIA, the search for variable objects and transient events is rapidly accelerating. There are, however important existing data-sets from instruments not originally designed to find such events. One example of such an instrument is the Solar Mass Ejection Imager (SMEI), an all-sky space-based differential photometer which is able to produce light curves of bright objects $(m \leqslant 8)$ with a 102 -minute cadence. In this paper we discuss the use of such an instrument for investigations of novæ, and outline future plans to find other variable objects with this hitherto untapped resource.
\end{abstract}

Keywords. stars: novæ, cataclysmic variables; astronomical data bases: miscellaneous; instrumentation: photometers

\section{Introduction}

The Solar Mass Ejection Imager (SMEI) is a high-precision white-light differential photometer (Buffington et al. 2006; Buffington et al. 2007) on board the CoRIOLIS solar satellite, and has been in operation since January 2003 (Eyles et al. 2003). The instrument consists of three baffled CCD cameras, each with a $60^{\circ} \times 3^{\circ}$ field of view, combining to sweep out almost the entire sky every 102 minutes with each orbit of the spacecraft (Hick et al. 2007). The peak throughput of the instrument is at approximately $700 \mathrm{~nm}$, with a FWHM $\sim 300 \mathrm{~nm}$, and it can reliably detect brightness changes in point sources down to approximately $8^{\text {th }}$ magnitude.

SMEI was originally designed to map large-scale variations in heliospheric electron densities from Earth orbit by observing the Thomson-scattered sunlight from solar-wind electrons in the heliosphere (Jackson et al. 2004). In order to isolate the faint Thomsonscattered sunlight, the much larger white-light contributions from the zodiacal dust cloud, the sidereal background and individual point sources (bright stars and planets) must be determined and removed (Hick et al. 2007 give details of the method). Brightness determination of point sources is therefore a routine step in the SMEI data analysis. SMEI is thus capable of providing unparalleled light curves with 102-minute cadence for many bright variable objects, allowing valuable opportunities to explore their fundamental parameters. 


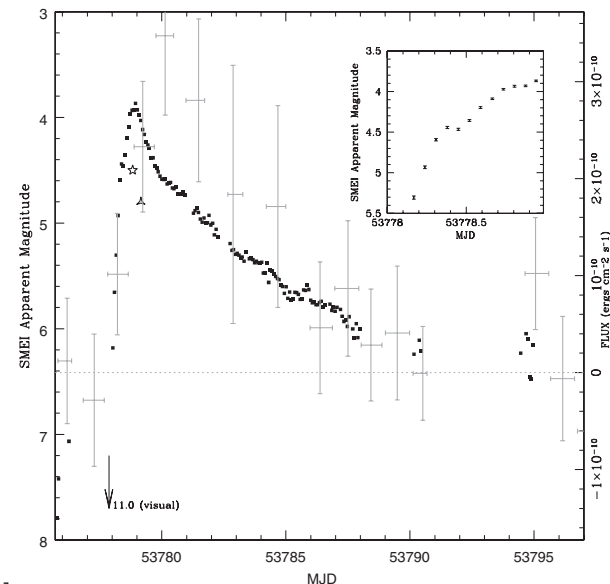

[a]

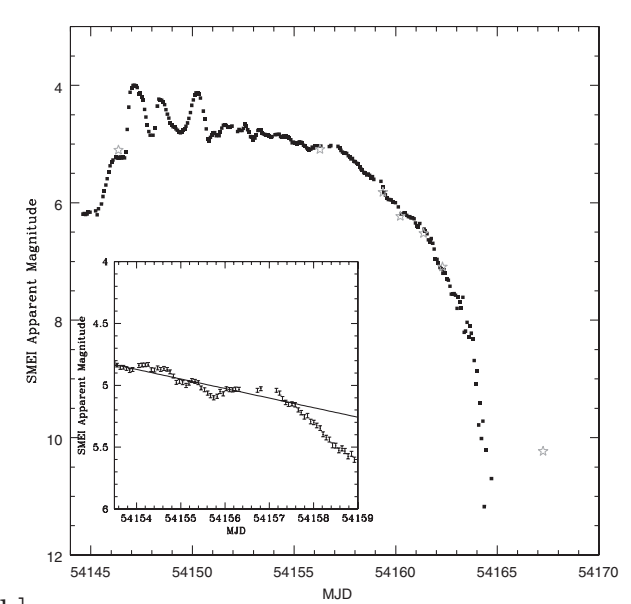

[b]

Figure 1. (a) SMEI light curve of RS Oph (black squares) in terms of "SMEI magnitude" (defined by Buffington et al. 2007) vs. time (left-hand y-axis). Over-plotted (grey) are the SwIFT BAT 14-25 keV data from Bode et al. (2006), right-hand y-axis. (b) SMEI light curve of V1280 Sco (black squares); superimposed (grey stars) are data from the " $\pi$ of the Sky" project. The inset shows the region around the light-curve break which is associated with the onset of dust formation. The solid line indicates the fit to the pre-break SMEI light curve and its extrapolation. Images have been reproduced by permission of the AAS from Hounsell et al. 2010, which includes more details for each plot.

\section{Examination of Novæ}

Previous analysis of the SMEI data set (Hounsell et al. 2010) focused on known bright novæ, in which three classical novæ (KT Eridani, V598 Puppis, V1280 Scorpii) and two recurrent novæ (RS Ophiuchi and T Pyxidis; see Hounsell et al. 2011 for the latter) were examined. The light curves produced by SMEI are unprecedented in their detail, and reveal (among other features) the epoch of the initial explosion, the reality and duration of any pre-maximum halt (found for all fast novæ in our sample, see Fig. 1b and Fig. 2a and b), the presence of secondary maxima (V1280 Sco, Fig. 1b), quasi-periodic variations (T Pyx, Fig. 3), the speed of decline of the initial light curve, plus precise timing of the onset of any dust formation (V1280 Sco, Fig. 1b). The SMEI data have also confirmed that two of the novæ (KT Eri and V598 Pup), which reached naked-eye brightness at peak, were undetected at that point and were only later re-discovered.

\section{Current and Future Work}

With approximately 8.5 years of all-sky photometry the SMEI archive contains a wealth of untapped data on many bright variable objects, many of which may be previously unidentified. Basing their work on an extrapolation of the observed nova density in M31, Darnley et al. (2006) have estimated a Galactic nova rate of $34_{-12}^{+15} y r^{-1}$, which is consistent with the previous estimate of $35 \mathrm{yr}^{-1} \pm 11$ derived by Shafter (1997). Of that subset Shafter (2002) estimates that roughly five novæ per year should reach $\mathrm{m}_{V}=8$ or brighter. However, in reality only one or two are actually detected, but nevertheless observations of many of those missed novæ must reside within SMEI data. To find such missing novæ a local point maximum search on four years' worth of SMEI data from camera 2 has been conducted. Initial results of the search have revealed the presence of over 1000 bright variable-object candidates, several of which are not found in the General 


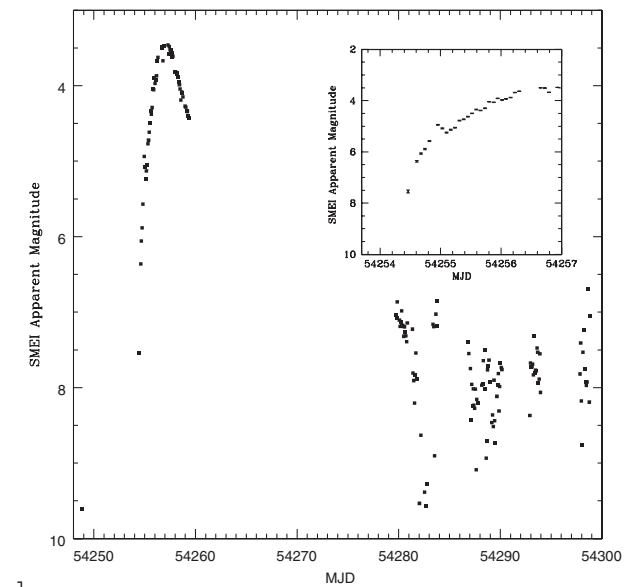
$[\mathrm{a}]$

Figure 2. (a) SMEI light curve of V598 Pup. The inset shows the rising portion of the light curve with an expanded time scale. (b) The black squares show the SMEI light curve of KT Eri, observed with Liverpool Telescope SkyCamT (LTSCT, described by Steele et al. 2004 and also in http://telescope.livjm.ac.uk/Info/TelInst/Inst/SkyCam/) data superimposed (grey stars). These images have been reproduced by permission of the AAS from Hounsell et al. 2010, which also gives more details for each plot.

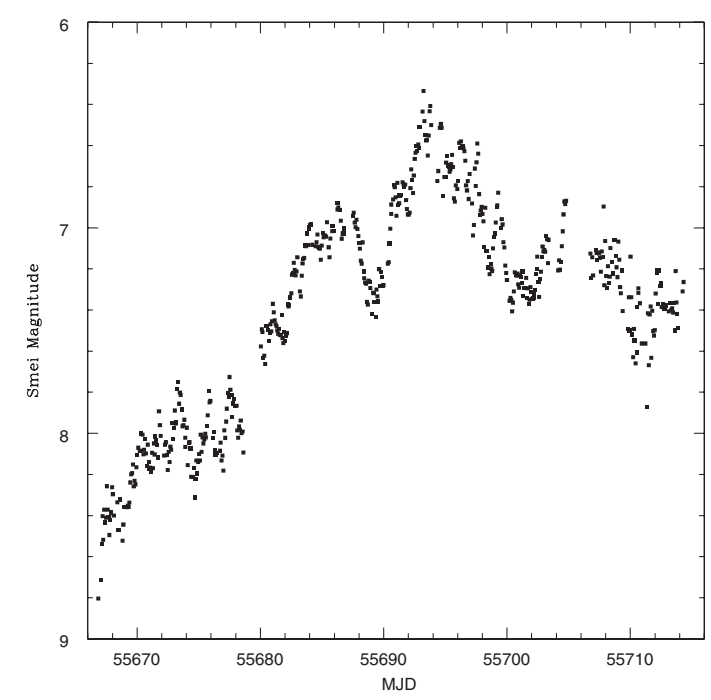

Figure 3. SMEI light curve of the 2011 outburst of T Pyx. The light curve clearly shows the quasi-periodic variations, and ends as the nova approached the Sun-wards exclusion zone (see Hounsell et al. 2011 for further details).

Catalogue of Variable Stars. We are currently conducting a search of the remaining years of data from all SMEI cameras. We hope to classify all variables found whilst also looking for novæ and other transients amongst them.

This work has demonstrates the importance of data sets like SMEI, and what a wealth of information can (unexpectedly) be held within such archives. SMEI data by themselves can thus provide significant information about many bright variables, but in combination with other all-sky data sets such as STEREO and potentially GAIA, SMEI can become an even more powerful tool. 


\section{Acknowledgements}

The USAF/NASA SMEI is a joint project of the University of California San Diego, Boston College, the University of Birmingham (UK), and the Air Force Research Laboratory. The Liverpool Telescope is operated on the island of La Palma by Liverpool John Moores University in the Spanish Observatorio del Roque de los Muchachos of the Instituto de Astrofisica de Canarias with financial support from the UK Science and Technology Facilities Council. We thank Gerry Skinner for provision of the BAT data on RS Oph and pointing us to the AFOEV observations of the outburst. P.P. Hick, A. Buffington, B.V. Jackson, and J.M. Clover acknowledge support from NSF grant ATM-0852246 and NASA grant NNX08AJ11G. A.W. Shafter acknowledges support from NSF grant AST-0607682. R. Hounsell acknowledge support from STFC postgraduate studentships and the Royal Astronomical Society for providing a supportive grant.

\section{References}

Bode, M. F., et al. 2006, ApJ, 652, 629

Buffington, A., et al. 2007, SPIE, 6689,

Buffington, A., Band, D. L., Jackson, B. V., Hick, P. P., Smith, A. C., 2006, ApJ, 637, 880

Darnley, M. J., et al. 2006, MNRAS, 369, 257

Eyles, C. J., et al. 2003, Solar Phys, 217, 319

Hick, P., Buffington, A., Jackson, B. V., 2007, SPIE, 6689,

Hounsell, R., et al. 2011, ATel, 3373, 1

Hounsell, R., et al. 2010, ApJ, 724, 480

Jackson, B. V., et al. 2004, Solar Phys, 225, 177

Shafter, A. W., 1997, ApJ, 487, 226

Shafter, A. W., 2002, AIPC, 637, 462

Steele, I. A., et al. 2004, SPIE, 5489, 679 